\title{
Photochemical Release of ATP from "Caged ATP" Studied by Time-Resolved Infrared Spectroscopy
}

\author{
Andreas Barth, ${ }^{\dagger}$ Karin Hauser, ${ }^{\dagger}$ Werner Mäntele, ${ }^{*,+, \neq}$ John E. T. Corrie, ${ }^{8}$ and \\ David R. Trentham ${ }^{8}$
}

Contribution from the Institut für Biophysik und Strahlenbiologie, Universität Freiburg, Albertstrasse 23, 79104 Freiburg, Germany, and National Institute for Medical Research, The Ridgeway, Mill Hill, London NW7 1AA, UK

Received March 28, $1995^{\circledR}$

\begin{abstract}
Rapid scan Fourier transform infrared (FTIR) spectroscopy and time-resolved single wavelength infrared (IR) spectroscopy have been used to follow the photochemical release of adenosine 5'-triphosphate (ATP) from $P^{3}$-(1-(2-nitrophenyl)ethyl) adenosine $5^{\prime}$-triphosphate (caged ATP). Vibrational difference spectra for the formation first of the aci-nitro anion intermediate and subsequently of ATP and the byproduct(s) were obtained by rapid scan FTIR spectroscopy in the millisecond-to-second time domain. Vibrational modes of the phosphate groups of ATP and caged ATP in the range $1250-900 \mathrm{~cm}^{-1}$ could be assigned on the basis of triple and single ${ }^{18} \mathrm{O}$ labeling in caged ATP at the terminal phosphate group and at the bridging oxygen between the terminal phosphate and the 1-(2-nitrophenyl)ethyl group, respectively. The rapid formation and subsequent decay of the aci-nitro anion intermediate were monitored by single-wavelength time-resolved $\mathbb{R}$ spectroscopy at $1251 \mathrm{~cm}^{-1}$ (predominantly a $\mathrm{PO}_{2}{ }^{-}$mode of caged ATP and the aci-nitro intermediate). The appearance of the free $\gamma$-phosphate group of ATP was monitored at $1119 \mathrm{~cm}^{-1}\left(\mathrm{PO}_{3}{ }^{2-}\right.$ mode of ATP). Decay of the aci-nitro anion intermediate and formation of ATP were well fitted by single exponentials to give a mean rate constant of $218 \pm 33 \mathrm{~s}^{-1}$ at pH 7 and $22{ }^{\circ} \mathrm{C}$.
\end{abstract}

\section{Introduction}

Rapid release of enzyme substrates or other biochemical effectors by flash photolysis of biologically inert but photosensitive precursors has become an established technique in the past decade. ${ }^{1}$ The 2-nitrobenzyl moiety has been widely used as a photosensitive protecting group but its photolysis byproduct, 2-nitrosobenzaldehyde, is highly reactive ${ }^{2}$ and damaging to biological preparations, ${ }^{3}$ so compounds substituted at the $\alpha$-carbon atom such as 1-(2-nitrophenyl)ethyl derivatives have been favored. ${ }^{3}$ The rate of release of the biologically active compound is a crucial aspect of the photochemistry as it relates to biological applications of these compounds and this process is generally controlled by dark reactions following the initial photochemical excitation and relaxation to a ground state. Aspects of these dark reactions, shown in Scheme 1, have been studied by several investigators ${ }^{1,4-7}$ and the $P^{3}$-(1-(2-nitrophe-

\footnotetext{
* Author to whom correspondence should be sent.

Universitat Freiburg.

¥ Present address: Institut für Physikalische und Theoretische Chemie, Universität Erlangen, Egerlandstrasse 3, 91058 Erlangen, Germany.

$\S$ National Institute for Medical Research.

$\otimes$ Abstract published in Advance ACS Abstracts, October 1, 1995

(1) (a) McCray, J. A.; Herbette, L.; Kihara, T.; Trentham, D. R. Proc. Natl. Acad. Sci. U.S.A. 1980, 77, 7237-7241. (b) McCray, J. A.; Trentham, D. R. Annu. Rev. Biophys. Biophys. Chem. 1989, 18, 239-270. (c) Kaplan, J. H. Annu. Rev. Physiol. 1990, 52, 897-914. (d) Adams, S. R.; Tsien, R. Y. Annu. Rev. Physiol. 1993, 55, 755-783. (e) Corrie, J. E. T.; Trentham, D. R. In Biological Applications of Photochemical Switches; Morrison, H., Ed.; Bioorganic Photochemistry Series, Vol. 2; Wiley: New York, 1993;
} pp $243-305$.

(2) (a) Patchornik, A.; Amit, B.; Woodward, R. B. J. Am. Chem. Soc 1970, 92, 6333-6335. (b) Amit, B.; Zehavi, U.; Patchornik, A. J. Org. Chem. 1974, 39, 192-196.

(3) Kaplan, J. H.; Forbush, B.; Hoffman, J. F. Biochemistry 1978, 17. $1929-1935$.

(4) (a) Schupp, W.; Wong, W. K.; Schnabel, W. J. Photochem. 1987 36, 85-97. (b) Zhu, Q. Q.; Schnabel, W.; Schupp, H. J. Photochem. 1987, $39,317-332$.

(5) Schneider, S.; Fink, M.; Bug, R.; Schupp, H. J. Photochem. Photobiol A $1991,55,329-338$.
Scheme 1
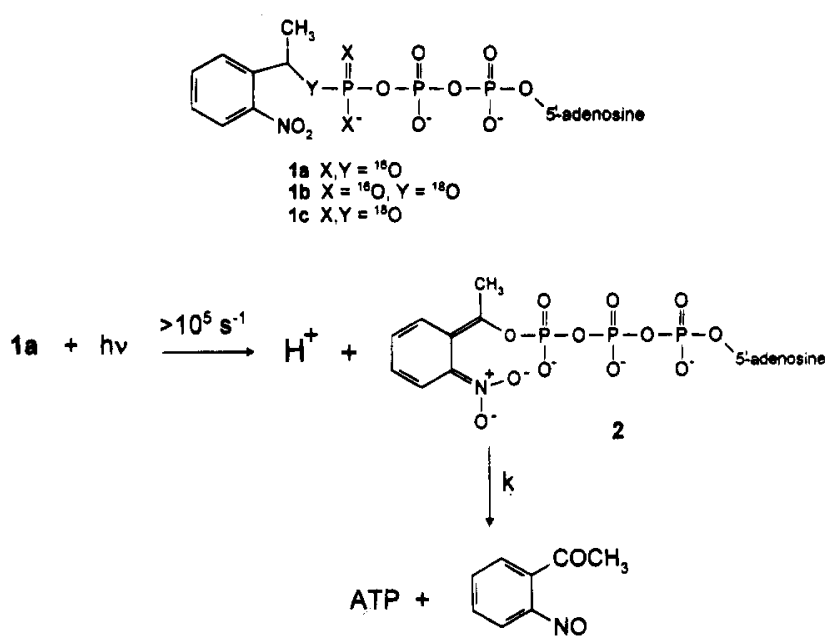

nyl)ethyl) ester of ATP, 1a, termed "caged ATP" has been of particular interest.

The rates of release of ATP or other biological substrates are generally deduced indirectly and are frequently inferred solely from the decay rate (rate constant $k$ in Scheme 1) of acinitro anion intermediates such as $\mathbf{2}$, which absorb in the nearUV. ${ }^{8,9}$ Although the decay of the aci-nitro anion 2 has been shown ${ }^{7}$ to be the rate-determining step for release of ATP, this need not necessarily be true for all caged compounds and the ability directly to monitor product release would be a valuable adjunct to the chemistry and biological application of these

(6) McCray, J. A.; Fidler-Lim, N.; Ellis-Davies, G. C. R.; Kaplan, J. H. Biochemistry 1992, 31, 8856-8861.

(7) Walker, J. W.; Reid, G. P.; McCray, J. A.; Trentham, D. R. J. Am. Chem. Soc. 1988, 110, 7170-7177.

(8) Walker, J. W.; McCray, J. A.; Hess, G. P. Biochemistry 1986, 25, 1799-1805.

(9) Corrie, J. E. T.; Katayama, Y.; Reid, G. P.; Anson, M.; Trentham, D. R. Phil. Trans. R. Soc. London A 1992, 340, 233-244. 
reagents. In contrast to the electronic spectra used to monitor aci-nitro decay rates, vibrational spectra are sensitive to localized molecular properties such as bond strengths, bond angles, and dipoles and can thus be used to follow photochemical transformations of a molecule in detail.

Time-resolved IR spectroscopy, used in conjunction with flash photolysis, will in principle permit precise monitoring of the chemical bond being cleaved or of the new group formed, i.e. the terminal phosphate group in the case of ATP. Various kinetic techniques for the infrared spectroscopic range have been developed for both Fourier transform infrared (FTIR) and dispersive spectrometers. ${ }^{10,11}$ Of the three FTIR methods, both the stroboscopic ${ }^{12}$ and step scan ${ }^{13}$ techniques require systems in which the process under study can be triggered in repetitive cycles. The rapid scan method, which at best has $c a .10 \mathrm{~ms}$ time resolution, is readily applicable to the investigation of the irreversible process of caged compound photolysis.

Time-resolved IR spectroscopy has been used previously in conjunction with flash photolysis to study reaction kinetics and structures of transient intermediates. Early work was concentrated on photolysis of metal carbonyls, where the intense carbonyl absorption provided a readily monitored signal. ${ }^{14}$ Recent papers have described studies on more complex molecules, including detection of a dehydroazepine intermediate from photolysis of phenyl azide ${ }^{15}$ and of an ynol from photodecarbonylation of phenylhydroxycyclopropenone, ${ }^{16} \mathrm{ki}$ netic studies on photogenerated radicals, ${ }^{17,18}$ and kinetic and product identification studies of the photolysis of 2-pyrone. ${ }^{19}$ In the majority of these examples, very high time resolution ( $\leq 1 \mu \mathrm{s}$ ) was attained but spectra could only be generated by sequential monitoring at a series of individual frequencies. The dark reactions which follow the photochemical events in caged compounds generally take place on the millisecond time scale which is well suited to FTIR spectroscopy. Non-time-resolved FTIR spectroscopy has previously been used to show features of the difference spectra between caged ATP and ATP. For example, appearance of a band at $1686 \mathrm{~cm}^{-1}$ due to the carbonyl bond of the byproduct (2-nitrosoacetophenone) was recorded. ${ }^{\text {le }}$ In a biological study of the mechanism of the $\mathrm{Ca}^{2+}$-transporting ATPase, in which enzymic activity was initiated by photolysis of caged ATP, the resulting difference spectra showed alterations in the nitro and $\mathrm{P}-\mathrm{O}$ modes ${ }^{20}$ as anticipated from the overall reaction in Scheme 1. Schneider et al. investigated the photolysis of several 2-nitrobenzyl and 1-(2-nitrophenyl)ethyl esters with time-resolved resonance Raman spectroscopy. 5 The technique has high time resolution and it was possible to observe

(10) Mäntele, W. Trends Biochem. Sci. 1993, 18, 197-202.

(11) Siebert, F. In Biochemical Spectroscopy; Sauer, K., Ed.; Methods in Enzymology, Vol. 246; Academic Press: New York, 1995; pp 501526.

(12) Braiman, M.; Ahl, P.; Rothschild, K. J. Proc. Natl. Acad. Sci. U.S.A. 1987, 84, 5221-5225.

(13) Uhmann, W.; Becker, A.; Taran, C.; Siebert, F. Appl. Spectrosc. $1991,45,390-397$.

(14) Ishikawa, Y.; Hackett, P. A.; Rayner, D. M. J. Am. Chem. Soc. 1987, 109, 6644-6650 and references therein.

(15) Li, Y. Z.; Kirby, J. P.; George, M. W.; Poliakoff, M.; Schuster, G. B. J. Am. Chem. Soc. 1988, 110, 8092-8098.

(16) Wagner, B. D.; Zgierski, M. Z.; Lusztyk, J. J. Am. Chem. Soc. 1994, $116,6433-6434$.

(17) Neville, A. G.; Brown, C. E.; Rayner, D. M.; Lusztyk, J.; Ingold, K. U. J. Am. Chem. Soc. 1989, 111, 9269-9270.

(18) Neville, A. G.; Brown, C. E.; Rayner, D. M.; Lusztyk, J.; Ingold K. U. J. Am. Chem. Soc. 1991, 113, 1869-1870.

(19) Amold, B. R.; Brown, C. E.; Lusztyk, J. J. Am. Chem. Soc. 1993, $115,1576-1577$.

(20) (a) Barth, A.; Kreutz, W.; Mäntele,W. FEBS Lett. 1990; 277, 147150. (b) Barth, A.; Mäntele, W.; Kreutz, W. Biochim. Biophys. Acta 1991, 1057, 115-123. (c) Barth, A.; Kreutz, W.; Mäntele, W. Biochim. Biophys. Acta 1994, 1194, 75-91. formation of the initial nitronic acid that subsequently, in polar solvents, ionized to an aci-nitro anion. However, the method is less sensitive than IR spectroscopy and gives less information about changes in the entire molecule than the FTIR method.

In the present study, the development of the flash photolysis technique coupled to time-resolved IR spectroscopy has centered on caged ATP, which provides a good paradigm to establish this approach since the kinetics of ATP formation upon flash photolysis have been determined by a variety of other but less direct methods. ${ }^{7}$ Those studies provided strong evidence that the aci-nitro anion intermediate 2 (Scheme 1) was rapidly formed and that its decay occurred concomitant with ATP formation. Characterization of the IR spectrum of the aci-nitro anion intermediate 2 is therefore an important aspect of the present work. Single or triple ${ }^{18} \mathrm{O}$ substitution of caged ATP as in isotopomers $\mathbf{1 b}$ and $\mathbf{1 c}$ provides a means to identify the IR bands associated with formation of the enol phosphate moiety of this intermediate, and of its subsequent decay with release of ATP. The experimental protocol involved two stages: Timeresolved FTIR difference spectra were first recorded in aqueous solution at $\mathrm{pH} 8.5$ and $1{ }^{\circ} \mathrm{C}$, where the aci-nitro intermediate is relatively long-lived. ${ }^{7}$ Positive and negative bands in these spectra were assigned to vibrational modes in caged ATP, the aci-nitro intermediate, and ATP. These assignments complement and extend the previous infrared study of ATP by Takeuchi et $a .^{21}$ Specific frequencies were then selected at which to follow the time course of the reaction under near-physiological conditions ( $\mathrm{pH} 7.0$ and $22^{\circ} \mathrm{C}$ ) with a dispersive IR spectrometer.

\section{Results}

FTIR Measurements. The photoinduced transition from caged ATP to ATP, in common with other 2-nitrobenzyl compounds, 1a,4,5,7 involves formation of a nitronic acid which rapidly ionizes to the aci-nitro anion 2 with release of a proton, followed by cleavage of the $\mathrm{C}-\mathrm{O}$ bond that links the 1-(2nitrophenyl)ethyl moiety to the $\gamma$-phosphate of ATP. At pH $8.5,200 \mathrm{mM}$ ionic strength, and $1{ }^{\circ} \mathrm{C}$, the decay of the acinitro anion occurs at a rate of $1 \mathrm{~s}^{-1}$ (estimated from the known activation energy and dependence on ionic strength). ${ }^{22}$ FTIR spectra recorded in the first $60 \mathrm{~ms}$ after the photolysis flash represent the transition from caged ATP to the aci-nitro anion 2 with $<5 \%$ decay of the latter (as calculated from the rate constant above), whereas spectra accumulated from 2.7 to 22.5 $s$ represent $>99 \%$ of the transition from caged ATP to the final products, i.e. ATP and the byproduct(s). Data collection was not extended beyond $22.5 \mathrm{~s}$ to minimize the influence on the spectra of possible slow reactions involving byproduct(s). For both transitions a difference spectrum was calculated, using the spectrum of caged ATP recorded prior to photolysis as the reference. Negative and positive bands in a difference spectrum arise from several sources. For example, disappearance or formation of a molecular feature in the course of the reaction leads to a negative band at the absorption frequency of the disappearing group and to a positive band for the newly formed group. Similar features are observed if the reaction results in a change of the extinction coefficient of a vibrational mode. A minimum (maximum) in the difference spectrum then indicates a reduced (increased) absorption of the reaction product as compared to the initial compound. Alternatively, a group may experience a shift in absorption frequency, leading to a pair of signals composed of a negative and a positive band. The negative band is characteristic for the absorption of the initial compound and the positive for the reaction product.

(21) Takeuchi, H.; Murata, H.; Harada, I. J. Am. Chem. Soc. 1988, 110, $392-397$.

(22) Reference 1b, p 255. 

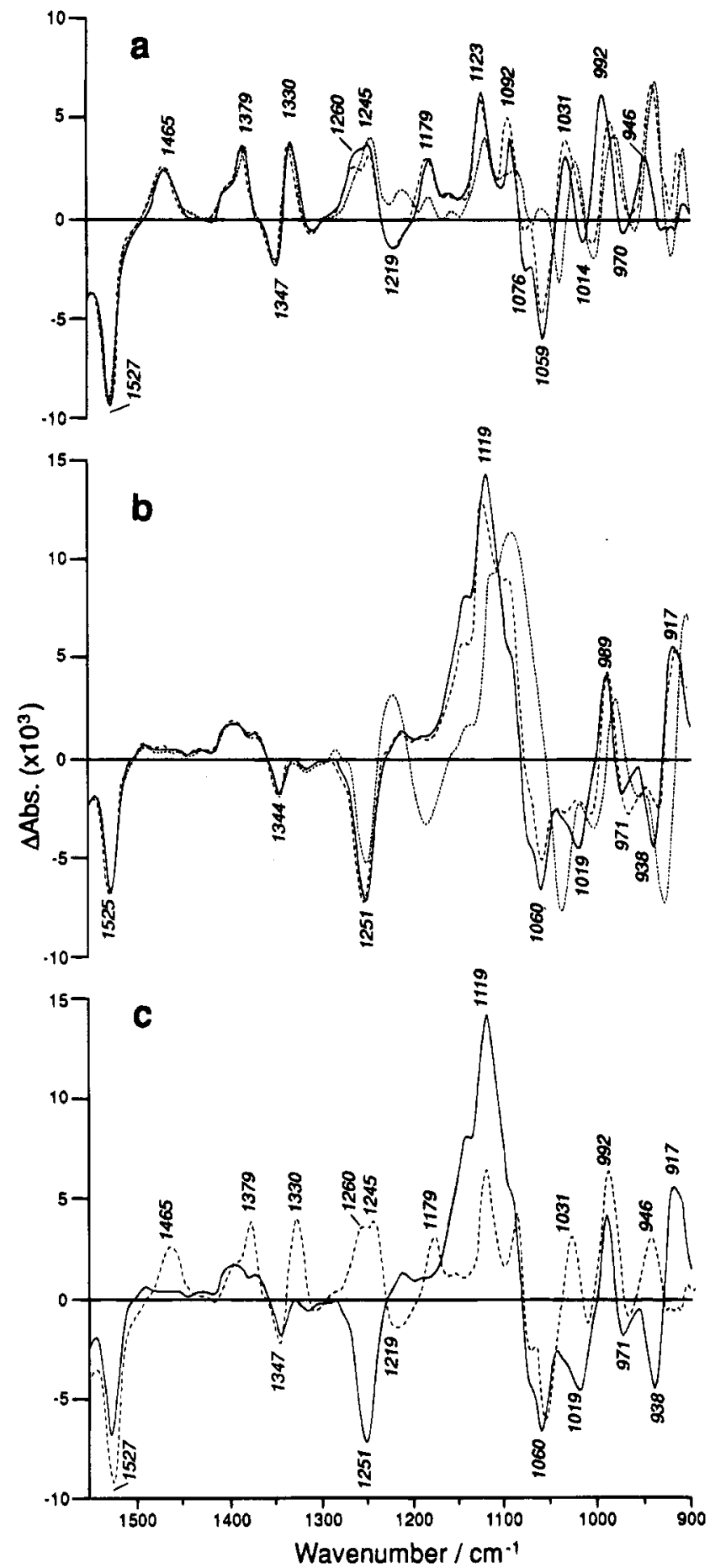

Figure 1. Infrared difference spectra of caged ATP photolysis, $1^{\circ} \mathrm{C}$, $\mathrm{pH}$ 8.5. (a) Spectra obtained $1-60 \mathrm{~ms}$ after the photolysis flash. Full line, compound $1 \mathbf{a}$; broken line, compound $\mathbf{1 b}$; dotted line, compound 1c. (b) Spectra obtained 2.7-22.5 s after the photolysis flash. Full line, compound 1a; broken line, compound 1b; dotted line, compound 1c. (c) Spectra for $\left[{ }^{16} \mathrm{O}\right]$ caged ATP 1a taken from parts (a) and (b). Broken line, from Figure 1a, full line, from Figure 1b. Spectra of different samples in parts (a) and (b) were normalized according to the difference band at $1525 \mathrm{~cm}^{-1}$. Labels in parts (a) and (b) refer to the full line spectra; labels in part (c) refer to the peak which is closest to the label.

The difference spectra for caged ATP photolysis are shown in Figure 1 for the frequency range $900-1550 \mathrm{~cm}^{-1}$. The highfrequency cutoff arises because proton release during the photolytic reaction causes a large change in the buffer absorption between 1550 and $1700 \mathrm{~cm}^{-1}$, which obscures other signals. The low-frequency cutoff arises from absorption by the $\mathrm{CaF}_{2}$ windows of the IR cell. The $\mathrm{H}_{2} \mathrm{O}$ solvent does not absorb in the spectral range shown.

Figure 1a shows difference spectra recorded in the first 60 $\mathrm{ms}$ after the photolysis flash for aci-nitro anion intermediate formation (i.e. absorption of aci-nitro anion minus absorption of caged ATP) from the caged ATP isotopomers 1a-c. The spectra are dominated by medium to strong positive bands which arise from the aci-nitro intermediate. The negative signals at 1527 and $1347 \mathrm{~cm}^{-1}$ have been assigned previously ${ }^{20 \mathrm{~b}}$ to the expected $^{23}$ antisymmetric and symmetric stretching modes of the nitro group which disappears from caged ATP upon the rapid formation $\left(>10^{5} \mathrm{~s}^{-1}\right)^{7}$ of the aci-nitro anion. Perturbations of the $\mathrm{P}-\mathrm{O}$ modes are expected in the aci-nitro intermediate, which is an enol phosphate, at frequencies below $1270 \mathrm{~cm}^{-1}$ and sharp difference signals are observed in this region. The ${ }^{18} \mathrm{O}_{3}$ labeling in compound 1c leads to significant changes from the ${ }^{16} \mathrm{O}$ difference spectrum, the most pronounced effect being the shift of the whole band profile below $1070 \mathrm{~cm}^{-1}$ to lower wavenumbers. This shift is also observed upon single isotope labeling (compound $\mathbf{1 b}$ ).

Figure $1 \mathrm{~b}$ shows difference spectra of the isotopomers $1 \mathrm{a}-\mathrm{c}$ for the overall transition from caged to free ATP (i.e. absorption of free ATP and byproduct(s) minus absorption of caged ATP). Apart from negative bands at 1525 and $1344 \mathrm{~cm}^{-1}$ as in Figure $1 \mathrm{a}$, the difference spectra are rather featureless in the range $1550-1280 \mathrm{~cm}^{-1}$. By contrast several strong bands appear in the range $1280-900 \mathrm{~cm}^{-1}$ that are significantly different in both frequency and amplitude on photolysis of the $\left[{ }^{18} \mathrm{O}_{3}\right]$ compound 1c compared to $\mathbf{1 a}$, but differ much less on photolysis of $\mathbf{1 b}$.

Figure $1 \mathrm{c}$ combines for $\left[{ }^{16} \mathrm{O}\right]$ compound 1a the difference spectra associated with the formation first of the aci-nitro anion and subsequently of free ATP, and provides a basis for selecting individual frequencies at which to monitor the ATP release kinetics. Notably, several bands specific to the aci-nitro intermediate (at $1465,1379,1330,1245$, and $1179 \mathrm{~cm}^{-1}$ ) are absent from the final spectrum of the end products, while new bands below $1300 \mathrm{~cm}^{-1}$ are generated upon release of free ATP. Assignments of relevant spectral bands are given in the Discussion section. These spectra show that the absorption changes at 1251 and $1119 \mathrm{~cm}^{-1}$ are relatively large in the transformations both of caged ATP to the aci-nitro anion 2 and of the anion to ATP and byproduct(s). Overall, as discussed in more detail below, the signal at $1251 \mathrm{~cm}^{-1}$ is associated with modification of the $\mathrm{PO}_{2}^{-}$vibration system and that at $1119 \mathrm{~cm}^{-1}$ with formation of the $\gamma-\mathrm{PO}_{3}{ }^{2-}$ group.

Single Wavelength IR Kinetics. The single wavelength recordings shown in Figure 2 were obtained at $\mathrm{pH} 7$ and 22 ${ }^{\circ} \mathrm{C}$. In each case a rapid process $\left(>10^{5} \mathrm{~s}^{-1}\right)$ is observed consistent with aci-nitro anion formation, followed by the ATP release step. The rates determined from fits to the kinetic traces, treated as single exponentials, were $200 \pm 32 \mathrm{~s}^{-1}$ at $1119 \mathrm{~cm}^{-1}$ and $244 \pm 12 \mathrm{~s}^{-1}$ at $1251 \mathrm{~cm}^{-1}$ (errors are standard deviations for $n=5$ ).

\section{Discussion}

The determination of the rate constant for ATP release from the data in Figure 2 depends on correct structural assignment of the bands at which the kinetics were measured and for this the use of oxygen isotopomers was essential. In addition, it is important to relate the IR spectral changes to the various species involved, i.e. caged ATP, the aci-nitro anion intermediate, free ATP, and byproduct(s). Since the present paper is focused on measurement of the ATP release rate, the following discussion

(23) Colthup, N. B.; Daly, L. H.; Wiberley, S. E. Introduction to Infrared and Raman Spectroscopy, 2nd ed.; Academic Press: New York, 1975. 


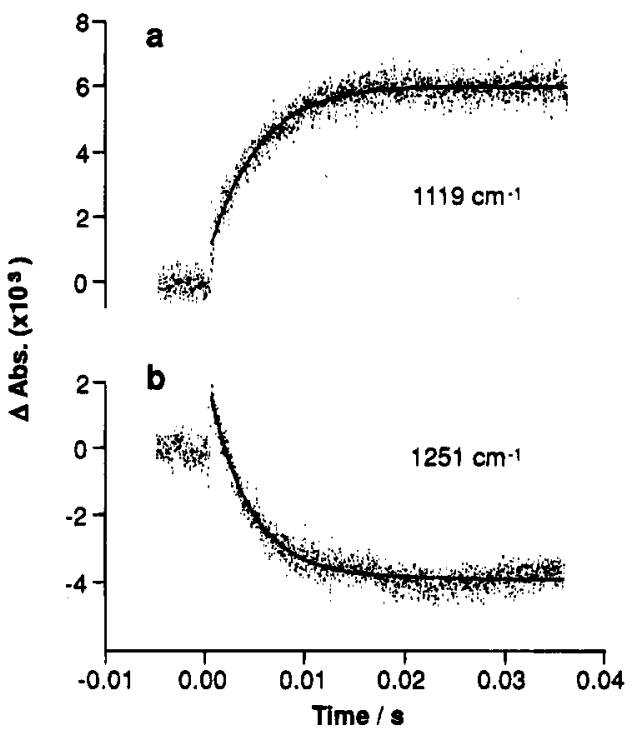

Figure 2. Kinetic IR absorption recording of caged ATP photolysis at (a) $1119 \mathrm{~cm}^{-1}$ and (b) $1251 \mathrm{~cm}^{-1}$ at $\mathrm{pH} 7$ and $22{ }^{\circ} \mathrm{C}$.

is principally confined to consideration of the phosphate modes, although other processes will also be occurring in the solution, e.g. formation of the byproduct (2-nitrosoacetophenone) and subsequent reactions thereof.

Transition of Caged ATP to the aci-Nitro Intermediate. For the spectra of the photolysis intermediate (Figure 1a) three distinct spectral regions can be distinguished. The range 1550$1300 \mathrm{~cm}^{-1}$ is unaffected by isotopic substitution and therefore includes no vibrations which involve the $\gamma$-phosphate group. The region 1300-1050 $\mathrm{cm}^{-1}$ shows several changes in band positions and intensities upon triple isotopic labeling but only slight differences for the $\left[{ }^{18} \mathrm{O}_{1}\right]$ isotopomer $\mathbf{1 b}$. Therefore this region must predominantly involve the nonbridging oxygen atoms of the $\gamma$-phosphate group. An exception occurs with the shoulder on the high-wavenumber side of the band at $1245 \mathrm{~cm}^{-1}$, which is reduced upon single isotopic labeling and further modified in the triple-labeled isotopomer. In the region 1020$900 \mathrm{~cm}^{-1}$ the band profiles of isotopomers $1 \mathbf{b}$ and $\mathbf{1 c}$ are shifted to the same extent with respect to the unlabeled species. Therefore the bridging oxygen atom, but not the nonbridging atoms, is involved in the corresponding vibrational modes. These are discussed in greater detail below.

The vibrational modes of the phosphate groups of caged ATP and the aci-nitro anion intermediate consist, by analogy to those of ATP in its trianionic form,,$^{21}$ of the antisymmetric $\left(\nu_{\mathrm{as}}\right)$ and symmetric $\left(v_{\mathrm{s}}\right) \mathrm{PO}_{2}^{-}$stretching vibrations at 1260-1200 and $1140-1050 \mathrm{~cm}^{-1}$, respectively, and the $\mathrm{P}-\mathrm{O}-\mathrm{P}$ modes below $1025 \mathrm{~cm}^{-1}$. In addition, a $\mathrm{C}-\mathrm{O}-\mathrm{P}$ mode would be expected for the bond between the $\gamma$-phosphate and the caging group.

The strongest band of the antisymmetric $\mathrm{PO}_{2}^{-}$modes in the absorption spectrum of caged ATP (data not shown) is centered at $1248 \mathrm{~cm}^{-1}$, with a shoulder near $1225 \mathrm{~cm}^{-1}$. In the difference spectra of formation of the intermediate (Figure 1a) a complicated band profile is observed in this region, comprising for unlabeled caged ATP a main positive band at $1245 \mathrm{~cm}^{-1}$, a positive shoulder at $1260 \mathrm{~cm}^{-1}$, and a negative band at 1219 $\mathrm{cm}^{-1}$. The position of the band at $1245 \mathrm{~cm}^{-1}$ is almost unaffected by isotopic substitution in the $\gamma$-phosphate so vibrations of this group do not contribute significantly to the band. Equivalent spectra from photolysis of other caged phosphates (not shown) also have a band at $1245 \mathrm{~cm}^{-1}$ that seems likely not to arise from $\mathrm{PO}_{2}^{-}$vibrations but has a small contribution from the bridging oxygen. Further isotopic labeling will be required to provide a definitive assignment. The spectral region adjacent to this positive band is clearly affected by isotopic labeling. The negative band at $1219 \mathrm{~cm}^{-1}$, which is found for unlabeled and single-labeled caged ATP $\mathbf{1 a}$ and $\mathbf{1 b}$, is not present for the triple-labeled compound 1c. Therefore this negative band can be assigned to a $v_{\mathrm{as}} \mathrm{PO}_{2}{ }^{-}$vibration of caged ATP to which the $\gamma$-phosphate group contributes. For the triple-labeled compound the negative band appears to be shifted by $\sim 40 \mathrm{~cm}^{-1}$ to $\sim 1180 \mathrm{~cm}^{-1}$, where it reduces the intensity of a positive band. While the assignment of this negative band is straightforward, that for the adjacent positive shoulder at $1260 \mathrm{~cm}^{-1}$ is less obvious. The shoulder is slightly reduced in intensity but barely shifted in frequency for the $\left[{ }^{18} \mathrm{O}_{1}\right]$ isotopomer $1 \mathrm{~b}$ and no clearly resolved band remains near this wavenumber in the triple-labeled isotopomer 1c. The residual asymmetry on the high-wavenumber side of the main band at $1245 \mathrm{~cm}^{-1}$ in the spectrum of isotopomer $1 \mathrm{c}$ seems likely to arise from minor perturbations of the $\alpha$ - and $\beta$-phosphate modes. While the nonbridging and bridging oxygens of the $\gamma$-phosphate are involved in vibrations which give rise to the $1260-\mathrm{cm}^{-1}$ band, the available data do not allow us to make a conclusive group-specific assignment.

The $\nu_{\mathrm{s}} \mathrm{PO}_{2}^{-}$vibrations of caged ATP absorb in the region $1140-1050 \mathrm{~cm}^{-1}$, with bands in the absorption spectrum (not shown) at $1131,1100,1076$, and $1059 \mathrm{~cm}^{-1}$. In the difference spectrum the negative band at $1059 \mathrm{~cm}^{-1}$ can be assigned to a $\nu_{\mathrm{s}} \mathrm{PO}_{2}{ }^{-}$vibration with a strong contribution of the $\gamma$-phosphate since it shifts to $1039 \mathrm{~cm}^{-1}$ upon triple isotopic labeling but is barely altered by single labeling. Strong positive bands at 1123 and $1092 \mathrm{~cm}^{-1}$ and the negative band at $1076 \mathrm{~cm}^{-1}$ are assigned to $\nu_{\mathrm{s}} \mathrm{PO}_{2}^{-}$vibrations which are however less localized on the $\gamma$-phosphate group, since they are less affected by isotopic labeling. The positions of these bands correspond closely to the values of 1123,1096 , and $1076 \mathrm{~cm}^{-1}$ reported ${ }^{21}$ for the $v_{\mathrm{s}}$ $\mathrm{PO}_{2}{ }^{-}$vibrations of ATP at $\mathrm{pH} 3$, where the terminal phosphate is singly protonated and hence resembles the monoalkylated terminal phosphate of caged ATP. Our results for triple labeling of the $\gamma$-phosphate parallel those of Takeuchi et al. ${ }^{21}$ who found that the two high-frequency modes are only slightly affected by labeling of the $\alpha$ - or the $\gamma$-phosphate group, whereas the lowest wavenumber component experiences a large $(20-30$ $\mathrm{cm}^{-1}$ ) downshift. The overall picture for the symmetric $\mathrm{PO}_{2}^{-}$ vibrations is a reduced spectral range of absorption upon formation of the aci-nitro intermediate arising from an upshift of the low-wavenumber components.

Finally, in the region below $1020 \mathrm{~cm}^{-1}$ the whole band profile is shifted $\sim 10 \mathrm{~cm}^{-1}$ to lower wavenumbers by both single and triple isotopic labeling. These bands are therefore associated with the $\mathrm{C}-\mathrm{O}-\left(\mathrm{PO}_{3}\right)_{3}-\mathrm{C}$ backbone modes that are perturbed by formation of the aci-nitro intermediate (Figure 1a). The shifts are smaller than expected for a localized $\mathrm{C}-\mathrm{O}$ vibration, suggesting coupling of the bridging $\mathrm{C}-\mathrm{O}$ mode with the $\mathrm{P}-\mathrm{O}$ modes, which are reported ${ }^{21}$ for ATP at 990 and $917 \mathrm{~cm}^{-1}$. This coupling prevents the assignment of a single band to the bridging $\mathrm{C}-\mathrm{O}$ bond of caged ATP.

Overall Transition from Caged ATP to Free ATP and Byproducts. Figure 1b shows spectra for formation of free ATP from caged ATP. As for the aci-nitro spectra, isotopic substitution has no effect in the spectral range $1550-1300 \mathrm{~cm}^{-1}$. However, it is of some interest to compare the intensities of the negative band at $\sim 1527 \mathrm{~cm}^{-1}$ in the spectra of the intermediate and final products (Figure 1c). At this wavelength there is no interference by overlap with other bands and the spectra consistently showed a higher intensity for this negative band in the intermediate than in the final spectrum. This implies that a proportion of the nitro groups which initially disappear 
through photochemical transformation to the aci-nitro intermediate may be regenerated in the course of its decay to the final products. It seems unlikely that the phenomenon is mediated simply by reversion to caged ATP, since the minimum of the band in the final spectrum is shifted by $2 \mathrm{~cm}^{-1}$ from its position in the intermediate spectrum, i.e. the regenerated nitro group does not absorb at the same wavenumber as it does in caged ATP. The possibility of an alternate decay path of the intermediate, which may not generate free ATP, will require further investigation.

In the region below $1300 \mathrm{~cm}^{-1}$ several bands can be assigned to phosphate vibrations which are not present in the spectra of the aci-nitro intermediate and are therefore associated with ATP liberation. Photolysis of $\mathbf{1 a}$ and $\mathbf{1 b}$ leads to a strong negative band at $1251 \mathrm{~cm}^{-1}$ which is reduced and modified in the adjacent spectral region in the spectrum of triple-labeled caged ATP 1c. Therefore we assign this band to antisymmetric $\mathrm{PO}_{2}{ }^{-}$ vibrations of caged ATP, which are reorganized in free ATP. The origins of this reorganization are 2-fold. First, the $\gamma-\mathrm{PO}_{2}{ }^{-}$ group of caged ATP is replaced by a $\mathrm{PO}_{3}{ }^{2-}$ group in ATP which absorbs at lower wavenumber. Therefore the absorption of the $\gamma-\mathrm{PO}_{2}{ }^{-}$group disappears, leading to a negative band in the difference spectrum in the region of the $\nu_{\mathrm{as}} \mathrm{PO}_{2}^{-}$absorption (1260-1200 $\left.\mathrm{cm}^{-1}\right)$. Second, the absorbance of the remaining $\alpha$ - and $\beta-\mathrm{PO}_{2}{ }^{-}$groups is shifted in free ATP $\left(1234 \mathrm{~cm}^{-1}\right)^{21}$ as compared to caged ATP $\left(1248 \mathrm{~cm}^{-1}\right)$, leading to a negative band at $\sim 1250 \mathrm{~cm}^{-1}$ and a positive band at $\sim 1230 \mathrm{~cm}^{-1}$ in the difference spectrum. These two contributions are best separated in the spectra of isotopomer 1c, since the isotopic substitution shifts the absorption of the $\gamma-\mathrm{PO}_{2}^{-}$group by $30-40 \mathrm{~cm}^{-1}, 21$ preventing overlap with the absorption of the $\alpha-$ and $\beta-\mathrm{PO}_{2}{ }^{-}$ groups. Therefore the disappearance of the triple-labeled $\gamma$-phosphate group upon free ATP formation is observed as a negative band at $1184 \mathrm{~cm}^{-1}$ ( $\nu_{\text {as }}$ for $\gamma-\mathrm{PO}_{2}^{-}$in triple-labeled caged ATP 1c) and the shift of the $\alpha-$ and $\beta-\mathrm{PO}_{2}{ }^{-}$absorption is revealed by the minimum at $1249 \mathrm{~cm}^{-1}$ and maximum at $1220 \mathrm{~cm}^{-1}$. For the isotopomers $1 \mathrm{a}$ and $\mathbf{1 b}$ the minimum from the antisymmetric vibrational mode of the $\gamma-\mathrm{PO}_{2}{ }^{-}$group in caged ATP and the maximum due to the change in the $\alpha$ - and $\beta$-phosphate absorption overlap at $\sim 1220 \mathrm{~cm}^{-1}$ and virtually cancel each other in the difference spectra. This simple explanation in terms of localized $\mathrm{PO}_{2}^{-}$group vibrations does not exclude the possibility of coupled $\mathrm{PO}_{2}{ }^{-}$modes.

The antisymmetric modes ${ }^{21}$ of the newly formed $\mathrm{PO}_{3}{ }^{2-}$ group lead to a broad structured band around $1120 \mathrm{~cm}^{-1}$ (Figure 1b), and this assignment is supported by a downshift of the center of the band by $\sim 20 \mathrm{~cm}^{-1}$ in compound 1c. For the singly labeled compound $\mathbf{1 b}$ this band shows a smaller shift, since the labeled oxygen becomes only one of three equivalent oxygen atoms in the $\gamma$-phosphate of ATP. The fine structure of this band is most likely due to the reorganization of the $\nu_{\mathrm{s}} \mathrm{PO}_{2}{ }^{-}$ modes that absorb in caged ATP as discussed above and in ATP (pH 7.5) at 1116 and $1087 \mathrm{~cm}^{-1} \cdot{ }^{21}$ The nearby negative band at $1060 \mathrm{~cm}^{-1}$ (assigned above to a $\nu_{\mathrm{s}} \mathrm{PO}_{2}{ }^{-}$mode) appears concomitant with formation of the aci-nitro intermediate and is therefore not connected with free ATP formation. In preliminary work $^{1 \mathrm{e}}$ where we examined only the difference spectra of the pre- and final post-photolysis states using caged ATP 1a and its triply labeled isotopomer 1c, this negative band was wrongly interpreted as being directly associated with a vibrational mode of the bridging $\mathrm{C}-\mathrm{O}-\mathrm{P}$ group. Use of the $\left[{ }^{18} \mathrm{O}_{1}\right]$ compound $\mathbf{1 b}$ in the present work has enabled us to correct this assignment.

The negative bands at 1019 and $938 \mathrm{~cm}^{-1}$ (Figure $1 \mathrm{~b}$ ) are assigned to backbone $\mathrm{C}-\mathrm{O}$ and $\mathrm{P}-\mathrm{O}$ vibrations of caged ATP, since they are shifted upon single isotopic labeling (Figure 1b and absorbance spectra, data not shown). However, the band at $938 \mathrm{~cm}^{-1}$ also seems to contain a contribution from nonbridging $\mathrm{P}-\mathrm{O}$ vibrations, since it is further shifted by the triple isotopic labeling. Two positive bands at 989 and 917 $\mathrm{cm}^{-1}$ that appear upon formation of free ATP were assigned by Takeuchi et al. ${ }^{21}$ to the $\mathrm{P}_{\alpha}-\mathrm{O}-\mathrm{P}_{\beta}\left(989 \mathrm{~cm}^{-1}\right)$ and $\mathrm{P}_{\beta}-\mathrm{O}-$ $P_{\gamma}\left(917 \mathrm{~cm}^{-1}\right)$ vibrations. However, they are affected by isotopic substitution in the $\gamma-\mathrm{PO}_{3}{ }^{2-}$ group, which indicates that the $v_{\mathrm{s}} \mathrm{PO}_{3}{ }^{2-}$ vibration contributes to these absorbance bands. The $989 \mathrm{~cm}^{-1}$ band shifts to $979-\mathrm{cm}^{-1}$ upon isotopic labeling of the three oxygen atoms of the $\gamma$-phosphate group (Figure 1b). If in addition the bridging $\beta, \gamma$ oxygen atom is labeled, ${ }^{21}$ this band is further shifted to $965 \mathrm{~cm}^{-1}$. The same shift of 14 $\mathrm{cm}^{-1}$ is observed upon labeling of the bridging $\alpha, \beta$ oxygen atom. ${ }^{21}$ We conclude that the ATP absorption at $989 \mathrm{~cm}^{-1}$ is due to a coupled vibration involving the $\nu_{\mathrm{s}} \mathrm{PO}_{3}{ }^{2-}$ vibration and $\mathrm{P}_{\alpha}-\mathrm{O}-\mathrm{P}_{\beta}-\mathrm{O}-\mathrm{P}_{\gamma}$ vibrational modes. By similar arguments we assign the $917-\mathrm{cm}^{-1}$ band to a coupled vibration that involves the same groups. Upon single and triple isotopic labeling of the $\gamma-\mathrm{PO}_{3}{ }^{2-}$ group, the band is shifted to 911 and $902 \mathrm{~cm}^{-1}$, respectively (Figure $1 \mathrm{~b}$ ). Additional labeling of the $\mathrm{P}_{\beta}-\mathrm{O}-\mathrm{P}_{\gamma}$ oxygen $^{21}$ shifts this band to $891 \mathrm{~cm}^{-1}$, which is a further shift of $11 \mathrm{~cm}^{-1}$ compared to labeling only of the three $\gamma$-phosphate oxygens. A shift of $16 \mathrm{~cm}^{-1}$ is observed upon labeling the $\beta$-phosphate group, including the $\mathrm{P}_{\alpha}-\mathrm{O}-\mathrm{P}_{\beta}$ oxygen. ${ }^{21}$ Therefore the $\nu_{\mathrm{s}} \mathrm{PO}_{3}{ }^{2-}$ vibration and a vibration of the whole $\mathrm{P}-\mathrm{O}-\mathrm{P}$ backbone leads to the absorption of ATP at $917 \mathrm{~cm}^{-1}$.

The analysis given above advances our understanding of the IR absorption bands of the triphosphate modes of ATP and its $P^{3}$-esters and provides valuable information for future IR measurements on biological systems that involve photolysis of caged nucleotides.

Single-Wavelength Kinetic Measurements. As the two bands at 1251 and $1119 \mathrm{~cm}^{-1}$ have been definitively assigned to specific phosphate vibrations associated with ATP liberation, they were suitable for directly monitoring the kinetics of this process with the dispersive IR spectrometer. At $1119 \mathrm{~cm}^{-1}$ there is an "instantaneous" increase in absorbance upon formation of the aci-nitro intermediate owing to a shift of absorption wavenumber of a $\nu_{\mathrm{s}} \mathrm{PO}_{2}^{-}$vibrational mode. Subsequent decay of the intermediate and liberation of ATP leads to an exponential further increase in absorption due to the newly formed $\mathrm{PO}_{3}{ }^{2-}$ group. At $1251 \mathrm{~cm}^{-1}$ the main contribution to the "instantaneous" increase in absorption is most likely due to a nonphosphate vibration. During the subsequent decay of the intermediate, there is an exponential decrease in absorption as this positive band disappears and a negative band develops that is due mainly to a shift in the absorption wavenumber of the $\alpha-$ and $\beta-\mathrm{PO}_{2}{ }^{-}$groups. The ATP release step has a measured rate constant of $218 \pm 33 \mathrm{~s}^{-1}$ averaged over all data recorded at 1251 and $1119 \mathrm{~cm}^{-1}$. The estimated value ${ }^{22}$ under these conditions of $\mathrm{pH}$ and ionic strength is $\sim 50 \mathrm{~s}^{-1}$. Two main factors may contribute to the discrepancy between the observed and estimated values. First, possible inaccuracy in the solution $\mathrm{pH}$ arising from manipulation of very small volumes of concentrated solutions would affect the rate, which is known to be $\mathrm{pH}$ sensitive. ${ }^{7}$ Second, the empirical relationship ${ }^{22}$ between rate constant and ionic strength may break down at the very high molarity of reagent and buffer used in these experiments. Future instrumental modifications will enable simultaneous monitoring of IR and UV signals, which are anticipated to give identical rate constants from the two measurements. Nevertheless, the principal goals of this study, i.e. to observe IR absorption changes associated with the 
$\mathrm{C}-\mathrm{O}-\mathrm{P}$ bond-breaking step leading to ATP generation and to show that the rate of ATP release is governed by the decay of the aci-nitro intermediate 2 , are unaffected by the numerical discrepancy in the present results.

Conclusion. While the spectral changes in the near-UV and visible spectra following flash photolysis of caged ATP follow single-exponential kinetics, a number of other caged compounds show complex time courses. Sometimes the decay of the putative aci-nitro anion is biphasic as in the case of 2-O-(2nitrobenzyl)glucose. ${ }^{24}$ With 3,4-dimethoxy-6-nitrobenzyl derivatives the UV spectrum of the putative aci-nitro anion is similar to that of the byproduct, making it difficult to be certain of the overall photolysis kinetics from visible absorption spectroscopy. ${ }^{25}$ For other compounds much slower visible absorption changes occur than have been claimed for release of the biological effector. ${ }^{\text {le }}$ Biological assays can validate such claims ${ }^{26}$ though the possibility that the aci-nitro anion or other intermediates are biologically active must not be overlooked. For these reasons, besides the intrinsic interest of 2-nitrobenzyl photochemistry, we suggest that the use of time-resolved IR spectroscopy in conjunction with flash photolysis will become an important tool for characterizing the photochemistry of caged compounds.

\section{Experimental Procedures}

Materials. Caged ATP 1a was prepared by esterification of ATP with 1-(2-nitrophenyl)diazoethane as previously described ${ }^{7}$ and the $\left[\gamma_{-18}{ }^{18} \mathrm{O}_{3}\right]$ analogue $1 \mathrm{c}$ was prepared similarly from $\left[\gamma_{-}{ }^{18} \mathrm{O}_{3}\right]$ ATP. ${ }^{27}$ The $\left[{ }^{18} \mathrm{O}_{1}\right]$ compound $1 \mathrm{~b}$ was prepared as described elsewhere. ${ }^{28}$ For the FTIR measurements, solutions contained caged ATP isotopomers 1a-c ( $85-90 \mathrm{mM}$ ) in $N, N$-bis[2-hydroxyethyl]glycine-KOH (Bicine) buffer (200 mM, pH 8.5). For the single-frequency kinetic measurements, solutions contained caged ATP 1a $(90 \mathrm{mM})$ in $N$-(2-acetamido)iminodiacetic acid-KOH (ADA) buffer $(800 \mathrm{mM}, \mathrm{pH} 7)$.

Spectroscopy. FTIR spectra were obtained on a modified Bruker IFS 66 spectrophotometer equipped with a mercury-cadmiumtellurium (MCT) detector of selected sensitivity. The Bruker OPUS software was used for rapid acquisition of spectra. Single-beam spectra were collected in time slots of $60-\mathrm{ms}$ duration, which were governed by the complete forward-backward movement time of the mirror in the interferometer for a spectral resolution of $4 \mathrm{~cm}^{-1}$. Difference spectra

(24) Corrie, J. E. T. J. Chem. Soc., Perkin Trans. 1 1993, 2161-2166.

(25) Wootton, J. F.; Trentham, D. R. In Photochemical Probes in Biochemistry; Neilsen, P. E., Ed.; NATO ASI Ser. C, Vol. 272; Kluwer: Dordrecht, The Netherlands, 1989; pp 277-296.

(26) Matsubara, N.; Billington, A. P.; Hess, G. P. Biochemistry 1992 $31,5507-5514$.

(27) Webb, M. R.; Trentham, D. R. J. Biol. Chem. 1981, 256, 1091010916.

(28) Corrie, J. E. T.; Reid, G. P. J. Labelled Compd. Radiopharm. 1995 , $36,289-300$ for the time intervals after the photolysis flash were calculated with respect to a single-beam spectrum consisting of 500 interferometer scans recorded immediately before the flash. Results for three separate samples were averaged. Photolysis of caged ATP was triggered by the OPUS software and achieved by a Xenon flash tube (N-185C, Xenon Corp., Woburn MA) mounted in the focal line of an elliptical mirror. A Schott UG11 filter was used to block the flash light in the infrared and visible part of the spectrum, resulting in a flash energy (at the area of the sample) of ca. $150 \mathrm{~mJ}$ in the spectral range 305$424 \mathrm{~nm}$. The sample cell consisted of a $\mathrm{CaF}_{2}$ window (20-mm diameter, $2-\mathrm{mm}$ thick) with a trough of $4-\mu \mathrm{m}$ depth and $8-\mathrm{mm}$ diameter. An aliquot of caged ATP solution was placed in the trough and the sample was sealed with a second flat window. This microcuvette holding less than $1 \mu \mathrm{L}$ was then mounted in a metal cell holder and thermostated to $\pm 0.5^{\circ} \mathrm{C}$.

The dispersive infrared spectrophotometer consisted of a mid-IR source (Ceramic Element No. 6575, Oriel, Stratford, CT), toroidal mirror optics of local design, a $0.5 \mathrm{~m}$ focal length monochromator (LOMO optics, St Petersburg, Russia, aperture f/3), an off-axis elliptical mirtor, and a semiconductor MCT detector (SN1299220-1, OEC GmbH, Zusmarshausen, Germany). Sample cuvettes, holders, and UV excitation were as for the FTIR experiments. Transient changes of the detector signal were amplified by a DC-coupled amplifier of local design and stored in a transient recorder (ADAM, TC2008-2, Maurer Instruments, Luzern, Switzerland) interfaced to a computer. Monochromator control, data transfer, and data treatment were done with the MSPEK program developed by S. Grzybek in W.M.'s laboratory. A detailed description of the spectrophotometer is given elsewhere. ${ }^{29}$ The measurements were performed at 1119 and $1251 \mathrm{~cm}^{-1}$ at a spectral resolution of $20 \mathrm{~cm}^{-1}$. For each wavenumber the IR signals of the first photolysis flash of 5 samples were recorded and averaged. A flash artefact, arising from transiently increased infrared emission of the sample, was superimposed on the kinetic records and decayed with a half-time of ca. $1 \mathrm{~ms}$. It was recorded after the first flash (with the measuring beam blocked) by averaging the signal from 10 further flashes and the resulting signal was subtracted from the signal recorded from the first flash.

Acknowledgment. We thank $\mathrm{H}$. Georg for his help in the initial phase of the project, S. Grzybek for assistance with data acquisition and analysis software, and $F$. von Germar for valuable discussions. We are grateful to Dr. M. R. Webb for the gift of $\left[\gamma-{ }^{18} \mathrm{O}_{3}\right]$ ATP. This work was supported by an ARC grant from the Deutscher Akademischer Austauschdienst and the British Council. W.M. acknowledges funding by the Deutsche Forschungsgemeinschaft (Ma 1054/10-1) and a Heisenberg Fellowship.

JA951024M

(29) Hauser, K. Diploma Thesis, Department of Physics, Albert-LudwigsUniversität, Freiburg, Germany, 1994; pp 27-52. 